\title{
Two-way microscale interactions between immigrant bacteria and plant leaf microbiota as revealed by live imaging
}

Running title: Microscale interactions within leaf microbiota

Shifra Steinberg ${ }^{1 *}$, Maor Grinberg ${ }^{1 *}$, Michael Beitelman $^{1}$, Julianna Peixoto ${ }^{1,2}$, Tomer Orevi $^{1}$, Nadav Kashtan ${ }^{1 \dagger}$

1 Department of Plant Pathology and Microbiology, Robert H. Smith Faculty of Agriculture, Food, and Environment, Hebrew University of Jerusalem, Rehovot, 76100 Israel

2 Current address: Laboratory of Enzymology, Department of Cellular Biology, Biological Sciences Institute, University of Brasilia, Brasilia, 70910-900, DF, Brazil

* These authors contributed equally to this work.

$\uparrow$ Corresponding author: Nadav Kashtan

email: nadav.kashtan@mail.huji.ac.i1

phone: +972-8-9489450

\begin{abstract}
The phyllosphere - the aerial parts of plants - is an important microbial habitat that is home to diverse microbial communities. The spatial organization of bacterial cells on leaf surfaces is non-random, and correlates with leaf microscopic features. Yet, the role of microscale interactions between bacterial cells therein is not well understood. Here, we ask how interactions between immigrant bacteria and resident microbiota affect the spatial organization of the combined community. By means of live imaging in a simplified in vitro system, we studied the spatial organization, at the micrometer scale, of the bio-control agent Pseudomonas fluorescens A506 and the plant pathogen $P$. syringae B728a when introduced to pear and bean leaf microbiota (the corresponding native plants of these strains). We found significant co-localization of immigrant and resident microbial cells at distances of a few micrometers, for both strains. Interestingly, this co-localization was in part due to preferential attachment of microbiota cells near newly formed $P$. fluorescens aggregates. Our results indicate that two-way immigrant bacteria - resident microbiota interactions affect the leaf's microscale spatial organization, and possibly that of other surface-related microbial communities.
\end{abstract}




\section{Introduction}

Leaf surfaces constitute a huge microbial habitat that is inhabited by diverse microbial populations including bacteria, yeast, and filamentous fungi (1-9). Bacteria are the most abundant organism among the leaf microbiota (3). The bacterial population of a typical leaf is comprised of hundreds of species of diverse phyla $(3,6,10)$. Bacterial cell densities on leaves may reach around $10^{5}-10^{6}$ cells per $\mathrm{cm}^{2}$, and cells are observed both as solitary cells and as aggregates that often comprise of several species $(3,11-13)$. The spatial organization of bacterial cells on the leaf surface is not uniform, and bacterial colonization correlates with the leaf's microscopic heterogeneity. Bacterial cells and aggregates have been shown to more likely colonize veins, trichome bases, stomata, and the cavities between epidermal cells $(1,8,11,14-16)$. It is thus clear that leaf microscale heterogeneity plays an important role in determining microbial organization on leaf surfaces. However, it is not well-understood whether, how, and at which spatial scales, cell-to-cell interactions affect the microscale spatial organization of leaf microbiota cells.

Mapping the spatial organization of the leaf microbiota population at micrometer and single-cell resolution is a challenge. Thus far, it has been done by means of microscopy of leaf inoculation experiments with a small number of interacting fluorescently-tagged model strains. This approach has revealed non-random dual-species spatial organization at the micrometer scale $(15,17)$. Other studies analyzed the natural microbiota on leaves using FISH probes $(13,18)$. These studies highlighted the complex interspecies interactions within natural leaf microbiota and shed light on the spatial scales of interspecies interactions, but were still limited to a low taxonomic resolution. The aforementioned studies suggest that interspecies interactions, at a micrometer spatial scale, are common on leaves. Yet, even if we could accurately map the microscale organization of cells on natural leaves, it would be hard to assess what is the net contribution of cell-to-cell interactions to the observed spatial organization, as leaf surface heterogeneity may mask this information.

Several studies have sought to understand the microscale colonization patterns of immigrant bacteria upon arrival to a new leaf, and how this organization affects cell survival. The fate of individual bacteria was found to depend upon variation in local conditions including e.g., nutrient concentrations and hydration conditions, that 
modulate the carrying capacity of microsites $(19,20)$. Moreover, aggregates of resident bacteria have been shown to facilitate the survival of immigrant bacteria cells that join them, on leaf surfaces $(21,22)$. The majority of these studies were not based on continuous live imaging, therefore they could not resolve whether cells preferentially attached to resident bacterial aggregates, or if cells that randomly attached near or onto aggregates had better survival rates.

Microscale interactions are also important for better understanding early colonization of pathogens and biocontrol agents. Little is known about the microscale interactions of an immigrant pathogen with the resident microbiota and their role in the pathogen's establishment. Because foliar pathogens typically spend some time as epiphytes before penetrating the leaf interior and causing disease, it is important to understand what they do and how they survive on the leaf surface. In addition, the early colonization of biocontrol agents is also of great interest. As these agents' establishment on the leaf is desirable, it is of interest to understand how interactions with the native microbiota affect their colonization.

In a previous study, we suggested that preferential attachment of cells to existing aggregates can improve survival in environments exposed to periodic stress (23). That work was based on an individual-based modeling approach that used computer simulations of foraging planktonic cells colonizing a surface under alternating wet-dry cycles. One of the questions we ask here is whether preferential attachment is observed experimentally.

This work examines how environmental leaf microbiota and artificially-introduced immigrant bacteria, spatially organize with relation to each other, post-inoculation. To focus on cell-to-cell interactions and to rule out the impact of leaf surface heterogeneity, we used a simplified experimental system based on glass-bottom multi-well plates. Two model strains were used as immigrant bacteria: Pseudomonas syringae B728a and $P$. fluorescens A506 (hereinafter: Ps B728a and Pf A506). Ps B728a is a foliar pathogen model strain that is the cause of brown spot on bean leaves (24-26). Pf A506 is a model bio-control agent that is used against Erwinia amylovora, the cause of fire blight disease in pears and apples $(27,28)$. These two strains were introduced to natural microbiota extracted from the surface of fresh leaves. Live imaging microscopy was used to track the microscale spatial organization of both immigrant bacteria and resident microbiota 
for up to 13 hours post inoculation. Analyses of the spatiotemporal organization of the combined population of the immigrant cells and the resident microbiota on the surface, at micrometer resolution, enabled us to study non-random mutual spatial organization patterns of immigrant cells and native microbiota.

\section{Materials and Methods}

\section{Experimental design}

A description of our experimental design appears in Fig. 1. Briefly, fresh leaves were picked from organically-grown, open-field green bean plants (Phaseolus vulgaris) and from an organically-grown pear tree (Pyrus communis) (Fig. 1A). The leaf surface microbiota of each plant was extracted as described below (Fig. 1C). In addition, to better represent the chemical environment of the leaf, leaf solutions were prepared for each plant species by blending fresh leaves (Fig. 1D). Fluorescently tagged Ps B728a or Pf A506 cells (Fig. 1B), which acted as the immigrant bacteria in our experiments, were inoculated into glass-bottom multi-well plates containing leaf solution media, with and without the natural resident microbiota extracted from bean and pear leaves, and surface colonization was tracked through live imaging (Fig. 1E-F).

\section{$\underline{\text { Strains and culture conditions }}$}

Pseudomonas syringae B728a (25) and Pseudomonas fluorescens A506 (28), isolated respectively from green bean and pear leaves, were kindly gifted by Steven E. Lindow, UC Berkeley. Ps B728a carried the pKT-TRP plasmid for a constitutive GFP expression (21), whereas Pf A506 was transformed using the $\operatorname{Tn} 7$ transposon system (29) to produce strains carrying an inserted gene cassette containing a constitutively expressed mCherry gene (this study). Cultures of Ps B728a and Pf A506 transformants were grown overnight in LB broth media, supplemented with either $30 \mathrm{mg} / \mathrm{ml}$ gentamicin or $50 \mathrm{mg} / \mathrm{ml}$ kanamycin respectively, under agitation of $400 \mathrm{RPM}$ at $28^{\circ} \mathrm{C}$. Subsequently, the strains were diluted in sterile phosphate-saline buffer (PBS 1X, 137 $\mathrm{mM} \mathrm{NaCl}, 2.7 \mathrm{mM} \mathrm{KCl}, 8 \mathrm{mM} \mathrm{Na}_{2} \mathrm{HPO}_{4}$, and $\left.2 \mathrm{mM} \mathrm{KH}_{2} \mathrm{PO}_{4}\right)$ and inoculated into 24well plates containing leaf solution only or leaf solution supplemented with natural leaf surface microbiota as described below.

\section{$\underline{\text { Leaf surface microbiota extraction }}$}


Fresh leaves from a pear tree and from green bean plants were sampled from organically grown plants (pear from Kiryat Malakhi area and bean from near Modi'in, Israel) in order to retrieve the microbiota associated with their surfaces. To extract the natural epiphytic microbiota from the leaf surface, we applied a protocol developed in our lab that is based on mechanical scraping. This method preserved most small and mid-size $(\sim 50 \mu \mathrm{m})$ aggregates intact, and efficiently recovered the majority of microbiota cells from the leaf surface. This method is an alternative method to the one proposed by Morris et al. that used agitation (30); while we found our method to yield qualitatively similar outcomes (i.e., extracted microbiota), the scraping methods seemed somewhat better at extracting more surface-attached aggregates, in keeping them intact, and in avoiding the possible bias introduced by filtration (see more about a comparison between the two methods in Supp. Fig. S1). Both the abaxial and adaxial sides of the leaves were individually scraped using a disposable cell spreader, after shallowly submerging the leaf in about $10 \mathrm{ml}$ of sterile PBS while ensuring that the petiole was not submerged in the solution. The scraped microbiota was then transferred to sterile $50 \mathrm{ml}$ Falcon tubes, and the remaining loosely attached microbes were re-suspended in about 1-3 $\mathrm{ml}$ of PBS from the post-scraping leaf. All leaves utilized in this experiment were picked within 12 hrs of scraping (and an additional 1 hour until microscope screens).

\section{$\underline{\text { Leaf solution }}$}

Leaf solutions of both pear and green bean leaves were prepared separately by blending 2 to 4 leaves, depending upon leaf size, in $500 \mathrm{ml}$ of PBS 1X. The resulting solution was then autoclaved and filtered through $0.2 \mu \mathrm{m}$ filters (Millex-GV) to remove leaf particles and intact microbial cells.

Microscopic analysis of the spatial organization of cells and microbiota on the surface Ps B728a and Pf A506 strains were grown overnight in LB broth media to a mid-log phase $\left(\mathrm{OD}_{600}=1.0,1 \mathrm{~cm}\right.$ path length $)$. The culture $\mathrm{OD}$ was first adjusted to $\mathrm{OD}=0.5$, and then further diluted by $1 \times 10^{3}$ in PBS (by employing serial dilutions). Leaf microbiota extractions (leaf wash) were diluted fivefold (pear) or tenfold (bean) to ensure that the microbiota density was not too high for the required spatial analysis (i.e., to ensure that the surface was not too densely covered by microbiota). Subsequently, $150 \mu 1$ of the leaf wash product containing the microbiota from either pear or bean 
leaves was gently inoculated into 24-well plates (24-well glass bottom plate \#1.5 highperformance cover glass - Cellvis, USA) containing $800 \mu 1$ of the corresponding leaf solution (i.e., pear or green bean leaf solution) per well. Experiments were done in triplicates (i.e., 3 wells per each immigrant and microbiota combination). Immediately after microbiota inoculation, the well plates containing the pear or bean systems were then inoculated with $50 \mu 1$ of diluted Pf A506 or Ps B728a. 24 well plates (lid on) were mounted on a stage top without warming (room temperature, $\sim 25^{\circ} \mathrm{C}$ ) during image acquisition.

Images were collected using an Eclipse Ti-E inverted epi-fluorescence microscope (Nikon, Japan) equipped with a Plan Apo 40x/0.95 NA air objective and the Perfect Focus System for maintenance of focus over time. An LED light source (SOLA SE II, Lumencor) was used for fluorescence excitation. $g f p$ fluorescence was excited through a 470/40 filter, and emission was collected with a T4951pxr dichroic mirror and 525/50 filter (filter cube \#49002, Chroma). mCherry fluorescence was excited through a 560/40 filter and emission was collected with a T5851pxr dichroic mirror and 630/75 filter (filter cube \#49008, Chroma). Images were acquired with a SCMOS camera (ZYLA 4.2PLUS, Andor Technology Ltd., UK) controlled with NIS Elements 5.02 software (Nikon Instruments Inc., USA). For time-lapse experiments, images were collected every $30 \mathrm{~min}$. At each time-point, $3 \times 3$ adjacent fields of views (covering a total area of $0.98 \mathrm{~mm}^{2}$ ) were monitored per each well. Multiple stage positions were collected using a motorized encoded scanning stage (SCANplus IM 130 x 85, Märzhäuse).

\section{$\underline{\text { Image processing and segmentation of microbial cells }}$}

The segmentation of native microbiota structures and immigrant bacteria cells on the surface was performed in two stages: First, the entire population on the surface was masked by Hybrid Range Filters, which identified objects in the bright field (BF) channel against the background (31). Second, each pixel of the objects was classified as either 'immigrant bacteria' or 'native microbiota' based on the intensity in both fluorescent channels and a manually calibrated piecewise-linear separator of RED vs GREEN channel intensities. To avoid misclassification, a margin of $1 \mu \mathrm{m}$ was defined around immigrant bacteria cells (or cell clusters), and any microbiota-labeled pixels in this border zone were removed from the segmentation. As the microbiota extraction method was not $100 \%$ free of plant-derived particles such as chloroplasts and tiny 
pieces of leaf epidermal cells, we further classified the microbiota 'class' into plantderived and microbiota-derived particles. We did this by manual classification, based on examination by eye of the overlay channels. While most plant-derived particles are characterized by their shape and auto-fluorescence, this was difficult to achieve algorithmically by a computer. We therefore decided to do this manually, twice, by two independent persons. By this means, between $0.4 \%-3.9 \%$ of the objects were classified as plant-derived particles (reflecting between $3 \%$ to $15 \%$ of the total area that was covered by particles on the well bottom). Overall statistics of plant-derived particles are presented in Supp. Table S1, and example images of classifications are provided in Supp. Fig S2.

\section{$\underline{\text { Spatial Analysis }}$}

Colonization pattern of immigrant bacteria in the absence of native micropbiota: Point correlation analysis was carried out by $2 \mathrm{D}$ dipole analysis (one-population, betweenobjects, whole ref. space) using DAIME software (32), and are shown in Supp. Fig. S3. Mean and 95\% confidence intervals (CI) were based on analyses of 9 sections $(3 \times 3$ tiles) of each image (image area is $0.98 \mathrm{~mm}^{2}$; one image was analyzed per well).

Co-localization of immigrant and microbiota cells or plant parts: Pair CrossCorrelation (PCC) and Nearest Neighbor (NN) analyses were carried out by 2D dipole analysis (two-populations, 500,000 random dipoles) and inflate analysis respectively, using DAIME software $(32,33)$. Mean and 95\% confidence intervals were based on analyses of 9 sections ( $3 \times 3$ tiles) of each image (image area is $0.98 \mathrm{~mm}^{2}$; one image was analyzed per well). Note that due to the classification process described earlier, it was necessary to use a narrow margin (of $1 \mu \mathrm{m}$ ) around immigrant cells to avoid misclassification of immigrants as microbiota. Thus, distances up to $1 \mu \mathrm{m}$ are interpreted as exactly $1 \mu \mathrm{m}$. Distance values over $1 \mu \mathrm{m}$ are unaffected by this artifact.

Alterative null random models of spatial organization: In addition to DAIME's pixelbased randomization null-model, alternative random models that preserve the size and shape of immigrant and microbiota cells and clusters were considered. (1) Objectrandomization, representing a random spatial organization, was produced by a Monte Carlo process: The reference population remained unchanged. All discrete objects in the analyzed population were picked in a random order, and each object (preserving its exact size and shape) was assigned a random location with no intersection with previous 
objects or with the reference population. The randomized datasets were analyzed using DAIME in a similar manner as the real data. Graphs in the relevant supplementary figures show the mean and $95 \%$ confidence interval of five different randomizations for each of the nine tiles (45 in total, per well). (2) label-randomized datasets, where microbiota and immigrant bacterial structures' labels were randomly permutated. Graphs in the relevant supplementary figures show the mean and $95 \%$ confidence interval of 100 different randomizations.

Aggregate-size distributions: In order to cluster tightly localized cells as aggregates, we performed morphological closing operations on the segmented masks with a structuring element of $2 \mu \mathrm{m}$ radius.

\section{Results}

We first performed surface colonization experiments with Ps B728a or Pf A506 cells in leaf solution only (i.e., without leaf microbiota cells, Fig. 1). Inoculation with bean leaf solution led to a more rapid and intense surface colonization of both strains than that with pear leaf solution (Fig. 2), possibly due to higher nutrient concentrations (consistent with growth curves in leaf solution in bulk, Supp. Fig. S4). Pf A506 cells tend to form aggregates on the surface, which grow over time. Typically, a nascent $P f$ A506 aggregate begins with the attachment of a single cell, and most of its progeny remains in the same location (see Supp. Figs. S5, S6). Ps B728a cells typically do not form pure aggregates, but rather colonize the surface more homogenously (see Supp. Figs. S5, S6). To test if the surface colonization pattern, with leaf solution but in the absence of microbiota, deviates from expected from random colonization, we performed pair-correlation spatial analyses (see Materials and Methods). These analyses showed that the spatial distribution of both Pf A506 and Ps B728a cells (or cell clusters) do not considerably deviate from the expected by chance (with one minor exception - see Supp. Fig. S3). These results also indicate that the surface is homogenous, and that there are no artifacts such as tiny submicron leaf pieces in the leaf solutions that might have attracted cells.

Next, we moved on to experiments with both immigrant and resident microbiota. The leaf microbiota extracted from both plants included bacteria, yeast-like cells, and fungi in both solitary and aggregated forms (as observed by microscopy, Fig. 3). Many of the aggregates were 'mixed', i.e., comprised of both bacteria and yeast cells. Microbiota of 
both leaves contained mostly smaller aggregates and fewer large ones of up to a few hundred $\mu \mathrm{m}^{2}$ (Fig. 3 and Supp. Fig. S7 E-H). To study the spatial organization of the combined population of immigrant and resident leaf microbiota, we tracked the respective surface colonization over time under the microscope (every 30 minutes for 13 hours). At each time point, we identified immigrant cells and all microbiota components through image analysis (Materials and Methods). Particles identified as plant derived were excluded from the analysis (Materials and Methods). As time passed from the inoculation point, an increasing number of both immigrant and microbiota cells were observed on the well bottom surface (Fig. 4, Supp. Fig. S7, S8-S11). Immigrant cells and microbiota cells were observed attaching to the surface, moving, dividing, detaching, and some of them forming cell clusters (micro-colonies) in the form of surface-attached aggregates. Of the microbiota cells, aggregates were observed sinking to the bottom surface (most aggregates sank over the first 2-3 hours, as might indicated from the temporal changes in aggregate-size distributions, Fig. S7 E-H), while some solitary cells were observed too, some of them clearly motile.

Next, we wanted to know whether and how the spatial organization of resident microbiota cells and immigrant cells on the surface was affected by their co-existence. More specifically we asked: Do immigrant cells and microbiota cells co-localize at the microscale more than expected by chance? To answer this question, we employed two commonly used, complementary methods for spatial analyses of our spatial data of the microscale organization of both immigrant and microbiota components on the well surface (Fig. 4 E-H). We used DAIME, a common tool for image and spatial analysis in microbial ecology (32), which implements both methods. The first method, termed pair cross-correlation (PCC), used DAIME 2D dipole algorithm (32-34); and the second method, termed nearest-neighbor $(\mathrm{NN})$, used DAIME 2D inflate algorithm $(32,33)$ (See Materials and Methods).

The overall picture, emerging from the analyses of the experiments of both immigrant strains, was that there were significantly more immigrant cells (or cell clusters) within a small distance, of a few micrometers, from resident microbiota components, than expected by chance (Fig. 5). The extent of deviation from random, and the spatial distances at which this deviation was observed, varied with method type, and between the four combinations of immigrant strain and plant species. Overall, qualitatively 
similar results were observed for three experimental replicates of each immigrantmicrobiota pair (Fig. S12).

At $\mathrm{t}=8 \mathrm{hrs}$ post inoculation, there were significantly more $P f$ A506 cells (or clusters) than expected by chance at distances $<\approx 5 \mu \mathrm{m}$ from resident pear microbiota, as reflected by both PCC and NN algorithms (Fig. $5,1^{\text {st }}$ row). In this type of analysis, at each given distance, values above 1 indicate co-localization of cells (immigrant and microbiota cells co-localize at this distance more than expected by chance) and values below 1 indicate that such co-localizations are less than expected by chance). In the presence of bean microbiota, the Pf A506 did not show deviation from random by PCC, but did show a clear deviation in the microbiota-to-immigrant $\mathrm{NN}$ analysis (Fig. 5, $2^{\text {nd }}$ row). Although Ps B728a showed no deviation from random based on PCC, it showed a clear deviation from random in the $\mathrm{NN}$ analyses when co-cultured with both pear and bean microbiota (Fig. 5, $3^{\text {rd }}$ and $4^{\text {th }}$ rows). Co-localization of Ps B728a to microbiota components was observed at distance $<\approx 2-3 \mu \mathrm{m}$ (Fig. $5,3^{\text {rd }}$ and $4^{\text {th }}$ rows). The differences in outcome of the PCC versus NN algorithms reflect differences in each algorithm's respective ability to detect existing spatial patterns and deviations from randomness of various datasets (see more in Discussion).

We further compared the results to those expected by chance based on several alternative null-models. In addition to the pixel-based null-model provided by DAIME, we performed randomization that preserves the size and shape of all immigrant cell clusters and microbiota entities detected on the surface, using Monte Carlo simulations (see Methods). We did it using two approaches: One that randomizes the localization of all immigrant cells clusters with and without allowing overlaps between objects (Fig. S13); and a second approach that randomly permutes the objects' labels (i.e., its classification as immigrant or microbiota object) (Fig. S14) (see Methods). Using these two stricter randomization models that take into account biologically relevant constraints that (a) preserve the size and shapes of microbial objects and (b) restrict spatial overlap between objects, increased the significance of co-localization at small distances, especially of the Dipole PCC algorithm (Fig. S14, S15).

Finally, we further asked if the observed co-localizations were due to one-way or twoway interactions. The asymmetry of the $\mathrm{NN}$ results, when comparing the two directions of the analysis, i.e., for which population the distances to nearest neighbor are computed 
(immigrant cells or microbiota; second and third columns in Fig. 5), led us to postulate that it tells us something about the underlying process that led to the observed colocalization. For example, whether these co-localizations resulted from preferential attachment of immigrant cells near microbiota cells, or vice versa. To further study this asymmetry, we used our live-imaging data to track all neighboring pairs of microbiota and immigrant bacteria (at distances of up to $2 \mu \mathrm{m}$ ), and checked which element of the pair was present earlier and which one joined subsequently. Interestingly, we found that for $P f$ A506, about $50 \%$ of co-localized pairs were a result of preferential attachment of resident microbiota cells to newly formed Pf A506 aggregates (Fig. 6A). This was also observed in time-lapse analysis, where it was easy to observe many cases of individual microbiota cells joining newly formed aggregates of Pf A506 (Fig. 6B, Supp. Files. S1, S2). This phenomenon is nicely reflected in the asymmetry of the NN analysis, in particular with $P f$ A506 and bean microbiota, where the microbiota-toimmigrant direction of the analysis showed a much stronger signal (reflecting solitary microbiota cells joining large $P f$ A506 aggregates) (Fig. 5). In contrast to $P f$ A506 cells, Ps B728a cells were not forming aggregates, and preferential attachment of microbiota cells to Ps B728a cells or cell clusters was not observed for either the native or nonnative plant microbiota (at least not within the first 9 hrs; Fig. 6A,B, Supp. Files S3,S4). Again, it was nicely reflected in the slightly stronger signal in the immigrant-tomicrobiota direction of the $\mathrm{NN}$ analysis (Fig. 5).

\section{Discussion}

In this work, we studied the spatial surface colonization pattern of cells of a co-culture of immigrant bacteria and natural resident leaf microbiota cells, through live imaging and spatial analyses at the microscale. We found that both the biocontrol agent $P f$ A506 and the plant pathogen Ps B728a display a significant co-localization pattern in cocultures of either native or non-native resident microbiota (pear and bean). Pf A506 colocalization was in part due to attachment of microbiota cells near newly formed $P f$ A506 aggregates, and Ps B728a showed a significant co-localization signal without such clear reciprocal preferential attachment.

The non-random co-localization of $P f$ A506 and $P s$ B728a with leaf microbiota, while not necessarily observable by the naked eye, was revealed by a rigorous image and spatial analysis. As our simplified system excludes the heterogeneity of natural leaf 
surfaces, this non-random organization must originate from interactions between the immigrant and resident microbiota cells. We found that native microbiota and immigrant bacterial cells may exhibit a spatial association, driven by biased attachment and movement on the surface. Our analyses indicate that most such interactions occur on the surface, and not in the bulk fluid: Most microbiota cells settle on the well surface within $2-3$ hours, and only $10 \%$ to $30 \%$ of co-localized immigrant-microbiota components arrived at the same time to the surface (Fig. 6 A). Indeed, detachment, reattachment, and movement of cells were previously suggested to play a role in the spatial organization of cells on leaf surfaces (35).

The spatial scales of interactions are of major importance in microbial ecology $(13,15$, 31, 36-38). Our work indicates that the effective scale for such co-localization is at distances of up to $\approx 5 \mu \mathrm{m}$, consistent with the conclusions of previous work on bacterial interactions on leaf surfaces $(13,15)$. In all four immigrant-microbiota combinations, about $50 \%$ (45-56\% at $8 \mathrm{hrs}$ ) of immigrant cells on the surface were at distances of up to $5 \mu \mathrm{m}$ from microbiota cells. Co-localization at distances above $\approx 5 \mu \mathrm{m}$ between immigrant and resident bacterial cells does not significantly deviate from random (Fig. 5) with the exception of a few cases of less-than-expected co-localizations (i.e., values below 1) at distances of 5-20 $\mu \mathrm{m}$ (Fig. 5, Fig. S15), possibly due to movement of cells within this distance range toward their counterparts.

We note that the various spatial analysis methods have different strengths and weaknesses, and that the differences in the detected deviation from random patterns between methods can be informative. For example, non-random patterns can be masked in PCC, if, for example, there is a big difference in the typical object size between the two populations, and distances are within the same order of scale (as can be seen in Supp. Fig. S3), which may explain the lack of deviation from random in PCC, while such deviation was detected by the $\mathrm{NN}$ algorithm. $\mathrm{NN}$ is limited in its ability to detect spatial patterns that are characterized by distances that are longer than the typical shortest distances between objects. As demonstrated in our results, the NN algorithm's asymmetry of results is also of interest, as it may reflect directionality or bias in the underlying process that generates the spatial organization (like the two-way interactions in our experiments). 
The choice of a proper meaningful random model for assessing spatial patterns, that takes into account the system's inherent properties and constraints is important. In particular, we suggest that the object-randomization model that we used, which preserves the size and shape of microbial objects (cells, clusters and aggregates) is important for the analysis of spatial data representing relatively sparse, noncontinuously populated spaces (e.g., bacterial colonization on water-unsaturated surfaces as opposed to saturated surfaces with continuous biofilms) and for uncovering patterns that are within the range of the objects' sizes, and of irregular shapes.

When examining the co-localization of immigrant bacteria and microbiota, comparison with object-based random models increased, in most cases, the significance of colocalization, particularly for the PCC 2D Dipole algorithm. This increase is mainly because microbial 'objects' sizes and irregular shapes add a constraint to the randomization - by prohibiting overlaps between objects - which is reflected in the apparent observed repulsion at distances less than, or comparable to, their size (see Supp. Fig. S15).

We note that our experiments were performed with leaf solutions that do not perfectly mimic the chemical environment on the leaf surface. Similarly to what bacteria face on natural leaf surfaces, the leaf solutions were nutrient poor, and contain compounds that microbes encounter on the leaf surface. They do, however, contain other compounds that originate from the leaf internals, including for example, vacuole components. Autoclaving the leaf solution could have also changed some of the solution properties. Because simple leaf washes (from leaf surface only) did not lead to the surface attachment of both studied bacterial strains, we made this compromise in order to study co-localization of immigrant and natural resident leaf microbiota in controlled, more tractable environments. In addition, we note that the extracted leaf microbiota include some plant-derived particles such as chloroplasts and tiny pieces of epidermis. However, as these were only small fraction of all microbiota components (by number and area), we believe that it did not have a major impact on the revealed observed twoway interactions. Our results were robust to the inclusion or removal of these from our analysis (data not shown). Interestingly, some indication that points to co-localization of Ps B728a to plant particles at distances of a few $\mu \mathrm{m}$ was also observed (Supp. Fig. S16). 
In a previous study, we used modeling and computer simulations to show that preferential attachment can be an efficient general surface colonization strategy for bacteria on 'patchily colonized' surfaces that are exposed to periodic stress such as desiccation, antibiotics, and predation (23). The present study provides experimental evidence for preferential attachment during surface colonization. This finding supports previous indications of preferential attachment in an in vitro experimental system using a single bacterial strain (39) or environmental microbiota (40). It is likely that biased surface colonization mediated by preferential attachment is a common bacterial organization trait within the phyllosphere, and possibly within other surface-related microbial habitats.

A non-random spatial organization such as the one that we observed, can be a result of various mechanisms. One example is the reported biased surface movement toward dense areas controlled by sticky polysaccharide trails (39). Differential hydrophobic cell-surface and cell-cell adhesion forces could also be at play (41). Other mechanisms may involve chemotactic movement toward aggregates (42), which may underlie the observed gravitation of $P S$ B728a toward microbiota aggregates; or informed attachment decisions, which may be the case in the observed association of Pf A506's and microbiota aggregates. Moreover, intra- and interspecies quorum sensing are possibly involved in such mechanisms $(42,43)$, especially as quorum-sensing signals on unsaturated surfaces such as plant leaf surfaces are highly localized, and quorum size can be surprisingly small, even as low as a few dozen cells $(42,44)$. Other sensing systems, such as peptidoglycan sensing by bacteria, can serve as indicators of cell proximity (45).

Regardless of the exact underlying mechanism, spatial organization may confer fitness advantage in environments that select for collective protection from stresses (23). Aggregates have been shown to increase bacterial cell survival on leaves under dry conditions $(21,46)$. In addition, aggregation has been found to increase survival on drying surfaces under moderate humidity through the formation of larger microscopic droplets around aggregates (47). These microdroplets have been shown to protect cells from desiccation (47). The association of immigrant bacteria and natural microbiota, while not necessarily mutually beneficial, may lead to increased protection. Finally, increased aggregate size may provide better protection from the various stresses that 
cells experience on leaf surfaces, including desiccation, UV radiation, antibiotics, and predation.

It is not clear if the association that we observed between immigrant cells and microbiota is specific to species or strains of the microbiota. As the leaf microbiota is comprised of hundreds of species, it is reasonable to assume that aggregates' specific species compositions affect spatial organization. Bacterial cells are known to sense their local environments, and cell colonization and behavior has been shown to be affected by interspecies signaling (48). Initial evidence for such interactions at the microscale, even between closely related strains within the same species, has been recently observed in isolated strains from the rhizosphere (49).

To conclude, our study demonstrates that inter-species microscale interactions likely play a role in the spatial organization of leaf microbiota. In particular, immigrant bacterial cells and resident microbiota tend to co-localize at the microscale, and this colocalization results from two-way interactions between immigrant and resident cells. These findings underscore the importance of individual-based approaches for improving of our understanding of leaf microbial colonization processes, with clear implications for plant pathology and the design of biocontrol approaches. More broadly, our results might be relevant to other surface-associated microbial habitats, including plant and animal microbiomes.

\section{Acknowledgements}

We thank Y. Hadar for valuable comments on the manuscript. We thank S. Lindow for kindly providing bacterial strains, and R. Feuchtwanger from Gan Hasadeh and N. Shachar for providing fresh leaves for this study. J. P. acknowledges the Lady Davis Trust for a postdoctoral fellowship. M. B. acknowledges the Rudin MSc scholarship. This work was supported by a research grant to N. K. from the James S. McDonnell Foundation (Studying Complex Systems Scholar Award, Grant \#220020475) and from the Israel Science Foundation (ISF \#1396/19). 


\section{Figure Legends}

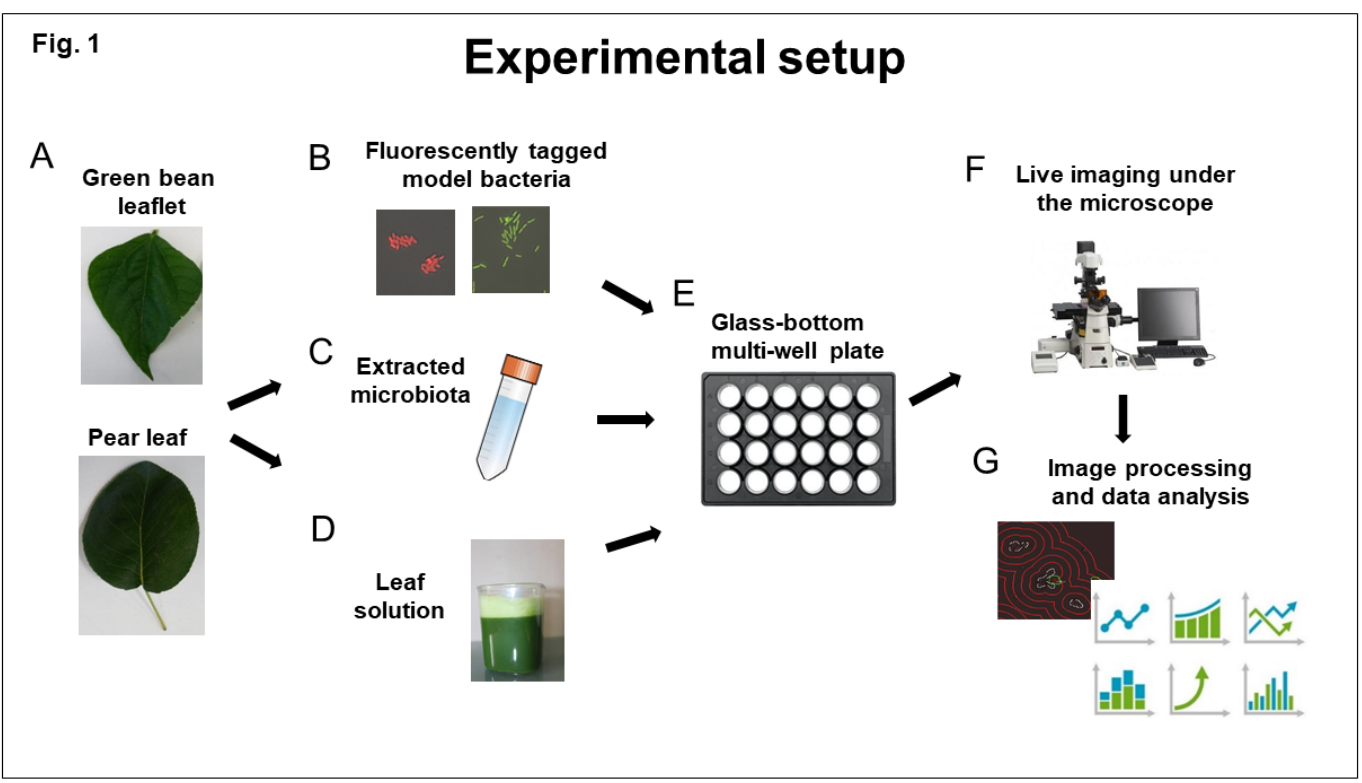

Fig. 1 Experimental Setup. A. Fresh green bean and pear leaves were picked. B. Fluorescently tagged $P s$ B728a and $P f$ A506 were used as immigrant bacteria. C. The natural microbiota were extracted from leaf surfaces D. Leaf solution was prepared from each plant. E. Ps B728a or Pf A506 were inoculated into glass-bottom wells with leaf solution only, or with both leaf solution and microbiota. F. Surface colonization was tracked over time under the microscope. G. Image processing and data analysis were performed using custom software in MATLAB and DAIME(32). 
Fig. 2

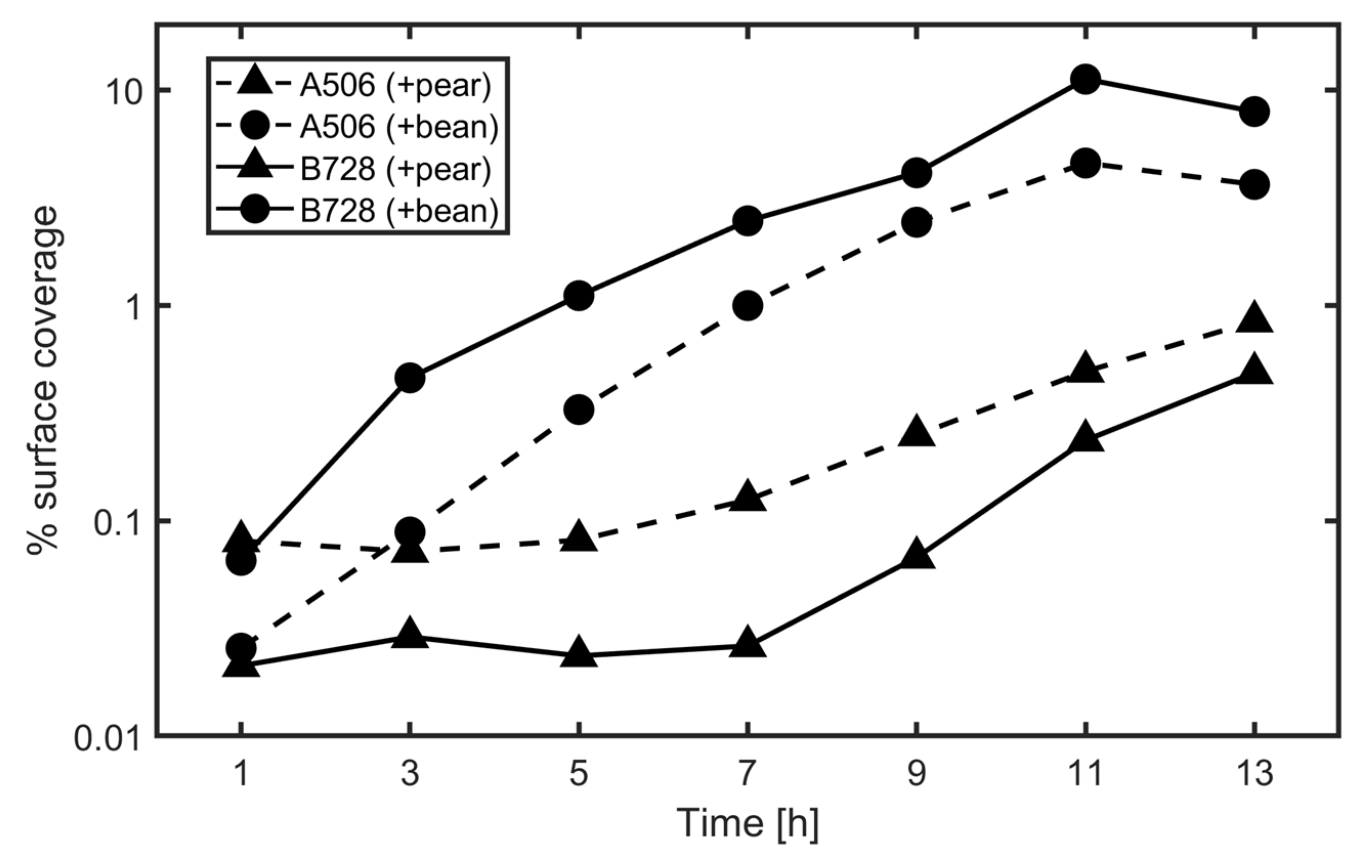

Fig. 2 Surface colonization dynamics without microbiota (leaf solution only). The graphs show the percentage of well surface covered by cells as a function of time. 


\section{Fig. 3}

A

\section{Bean microbiota}
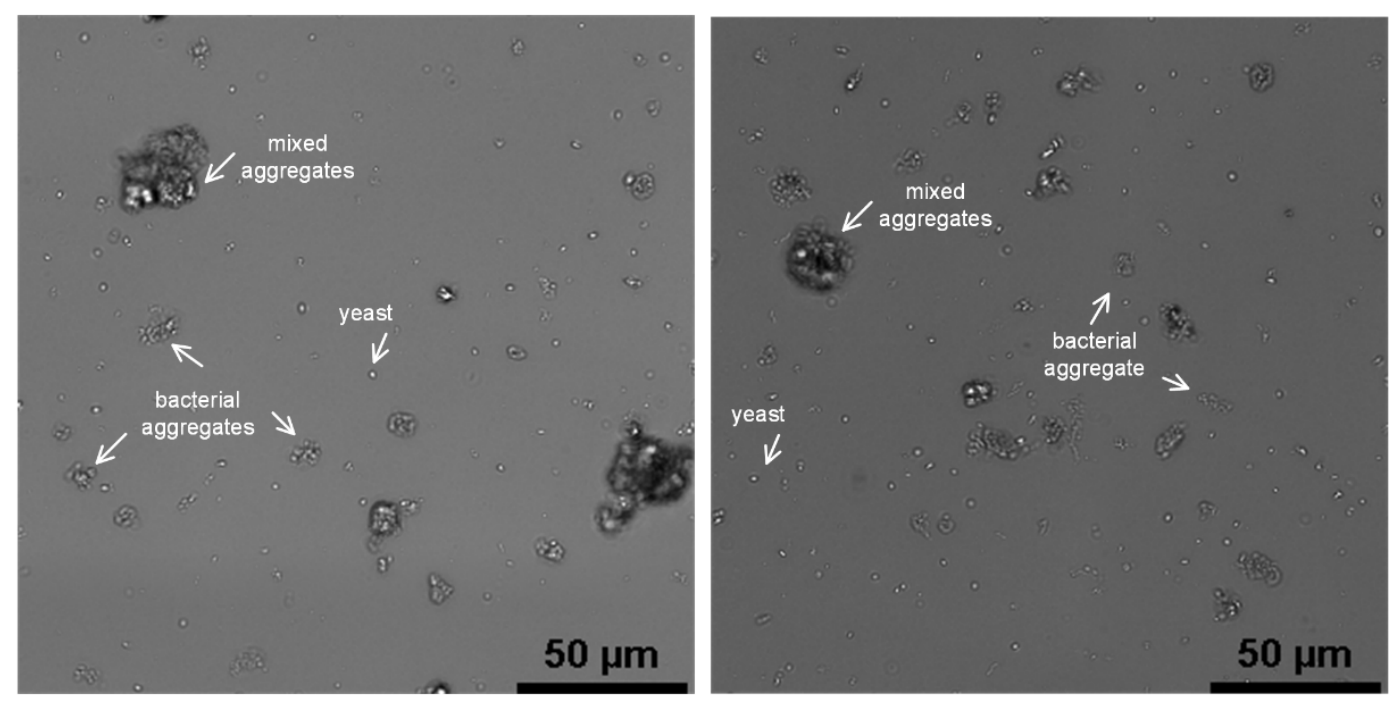

B

Pear microbiota
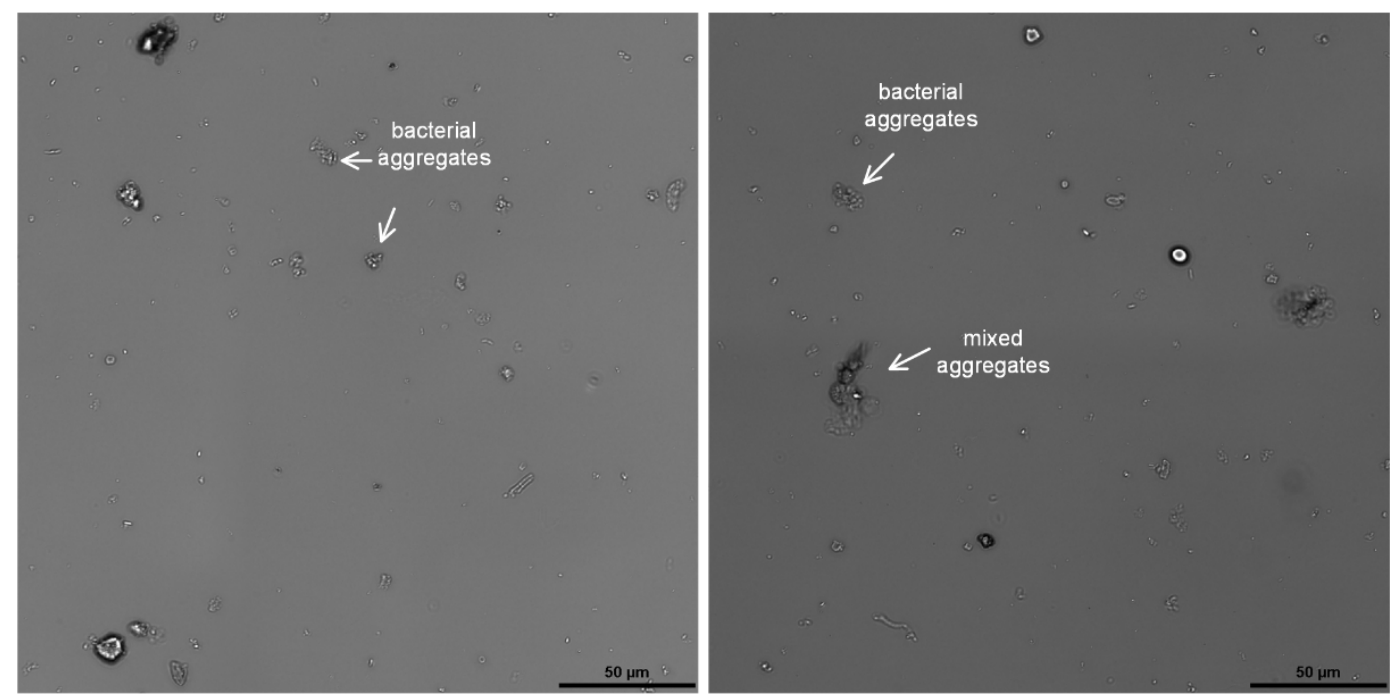

Fig. 3 Extracted microbiota from bean and pear leaves. Two representative images of the extracted microbiota from bean (A) and pear (B) leaf surfaces, as observed on glass-bottom well plates, prior to immigrant bacteria colonization. 
Fig. 4

Surface colonization of immigrant bacteria \& resident microbiota

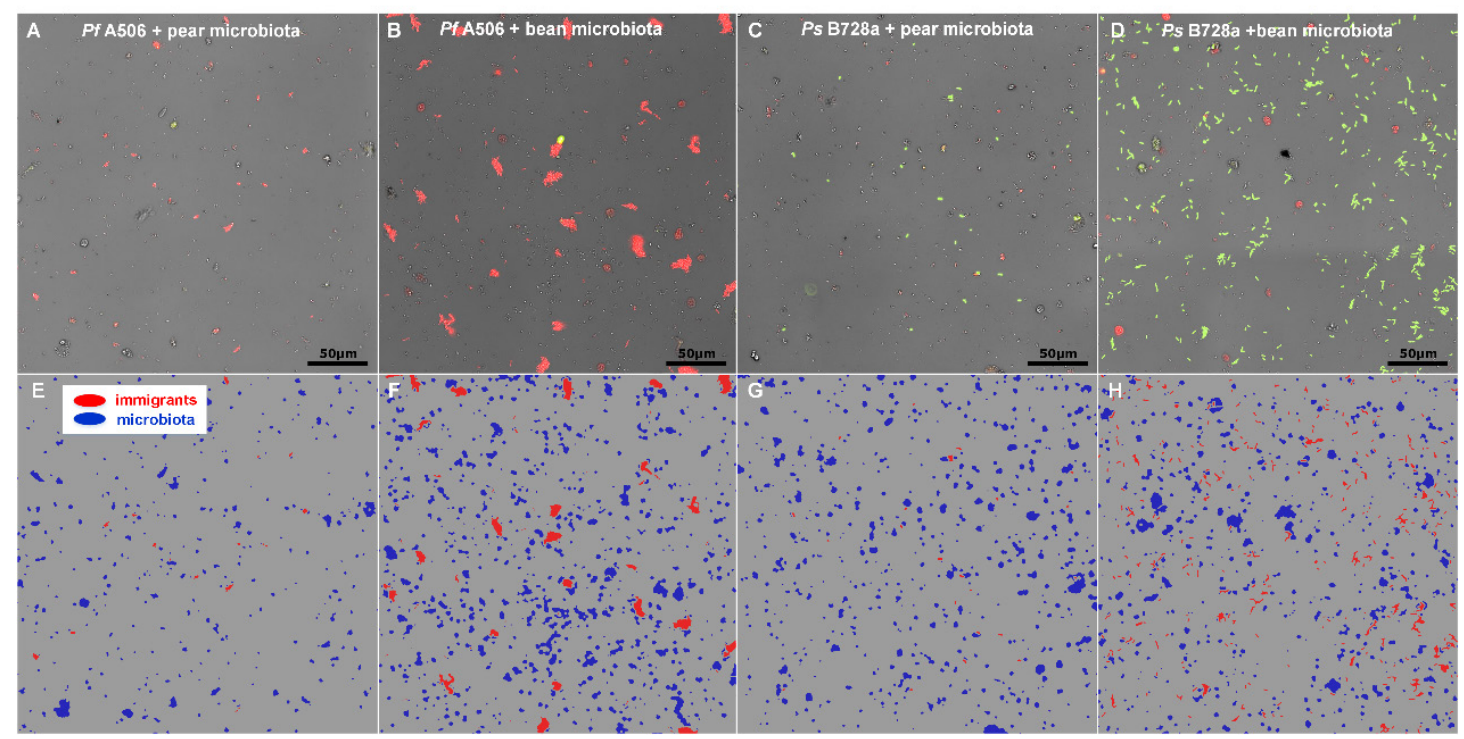

Fig. 4 Surface colonization of immigrant bacteria introduced to resident

microbiota. A. Pf A506 (red) with pear microbiota. B. Pf A506 (red) with bean microbiota. C. Ps B728a (green) with pear microbiota. D. Ps B728a (green) with bean microbiota. All images were taken $8 \mathrm{hrs}$ post inoculation. Note the differing colonization patterns of the two strains: Pf A506 formed aggregates, while Ps B728a remained mostly as solitary cells that colonized the surface in a less 'clustered' pattern (see also Supp. Fig. S7). E-H. The corresponding classification of the overall population into immigrant cells (red) and microbiota components (blue). Objects suspected as plant particles were removed. 
Fig. 5

Spatial analysis of combined immigrant bacteria and resident microbiota populations

Dipole
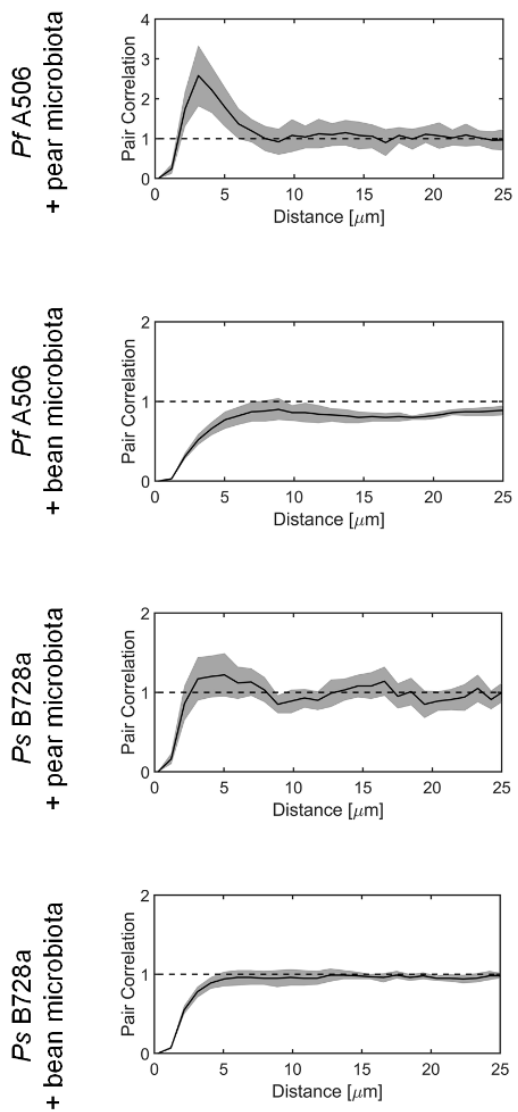

Inflate

(bacteria-to-microbiota)
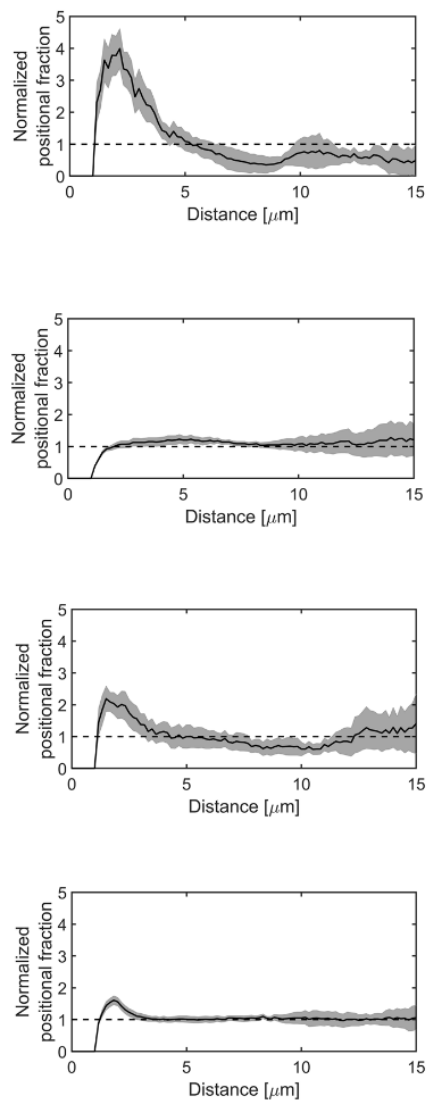
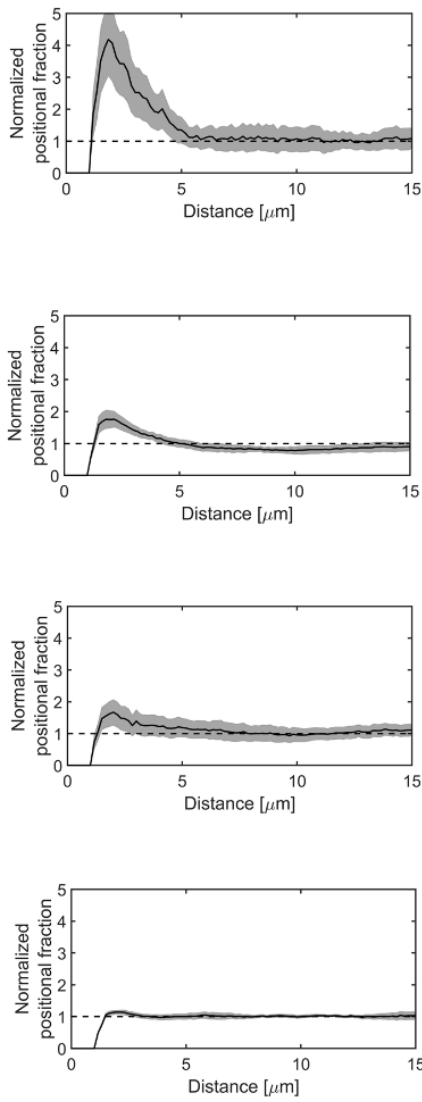

(microbiota-to-bacteria)

Fig. 5 Spatial analysis of combined immigrant bacteria and resident microbiota based on Pair Cross-correlation and Nearest Neighbor methods. Shown are results of pair cross-correlation (PCC), using DAIME 2D dipole algorithm; and nearestneighbor $(\mathrm{NN})$, using DAIME 2D inflate algorithm $(32,33)$ (See Materials and Methods). Black line and gray envelopes present mean and 95\% confidence intervals. In this type of analysis, deviation from the expected by chance are at distances significantly above or below a value of one. For all combinations of immigrant strain and plant microbiota, at least by one of the methods, there are significantly more cells at short distances to microbiota cells, than expected by chance. Note that when considering an alternative object-based randomization scheme (See Methods), the Dipole algorithm shows co-localization at distances of up to 5-10 $\mu \mathrm{m}$ (Supp. Fig. S15). Note that all minimal observed distances $<1 \mu \mathrm{m}$ are considered a distance of exactly $1 \mu \mathrm{m}$ (due to technical reasons, see Materials and Methods). 
Fig. 6

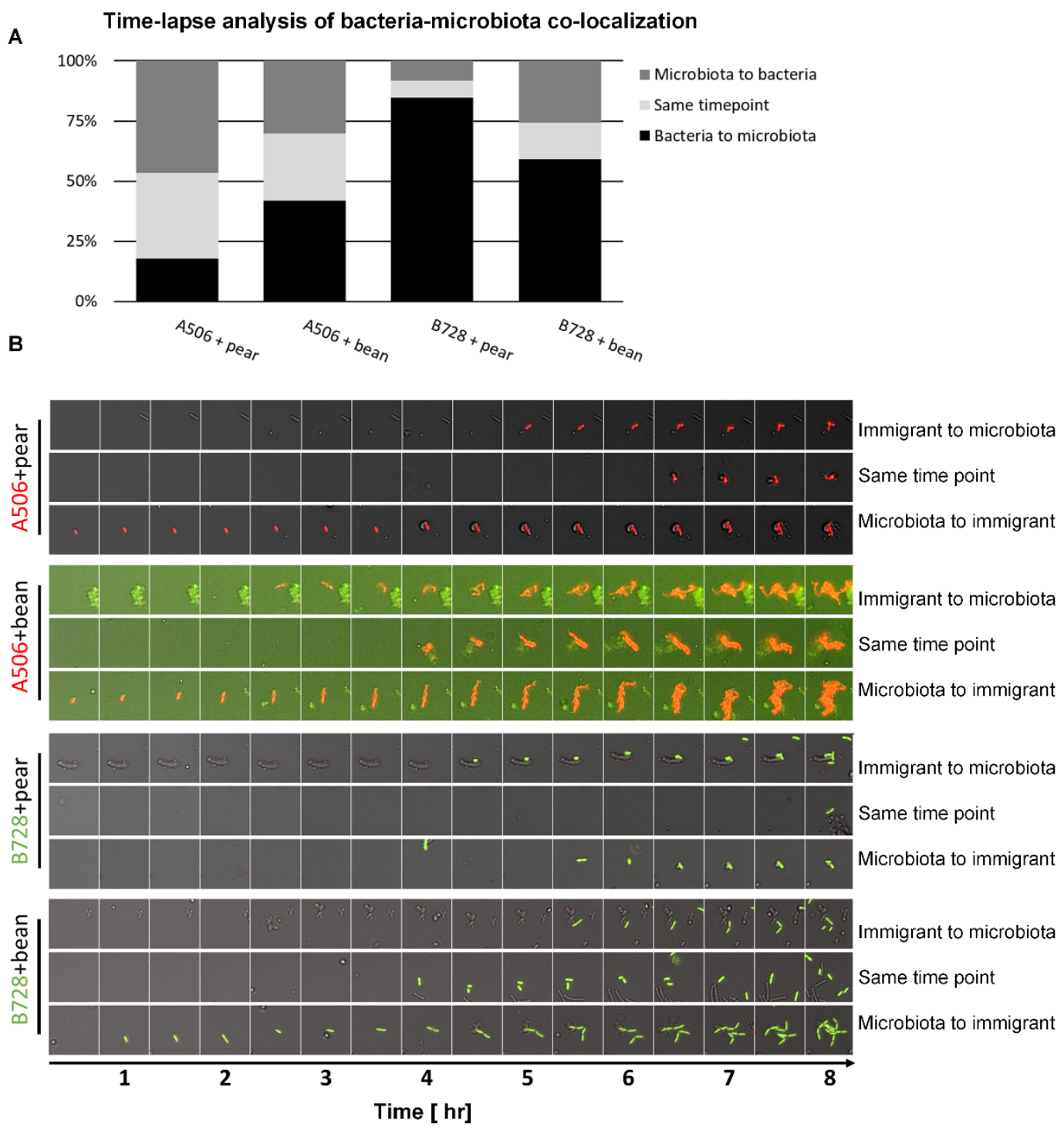

Fig 6. Two-way microscale interaction dynamics. Inspection of co-localized immigrant cells and microbiota shows incidences of all three possible scenarios: immigrant cells joining microbiota cells, microbiota cells joining immigrant cells, and both arriving at the same time point (same 30-minute time window) to the surface. A. The fraction of each of the three scenarios per each strain-microbiota pair (based on manual inspection of more than 100 randomly selected co-localized objects). B. Example time-series images of immigrant-to-microbiota attachments, same time-point, and microbiota-to-immigrant events (more time series are provided as Supp. Files 1-4). 


\section{References}

1. Lindow SE, Brandl MT. Microbiology of the phyllosphere. Appl Environ Microbiol. 2003;69(4):1875-83.

2. Lindow SE, Leveau JH. Phyllosphere microbiology. Current Opinion in Biotechnology. 2002;13(3):238-43.

3. Vorholt JA. Microbial life in the phyllosphere. Nature Reviews Microbiology. 2012;10(12):828.

4. Vacher C, Hampe A, Porté AJ, Sauer U, Compant S, Morris CE. The phyllosphere: Microbial jungle at the plant-climate interface. Annual Review of Ecology, Evolution, and Systematics. 2016;47:1-24.

5. Bringel F, Couée I. Pivotal roles of phyllosphere microorganisms at the interface between plant functioning and atmospheric trace gas dynamics. Frontiers in Microbiology. 2015;6:486.

6. Redford AJ, Bowers RM, Knight R, Linhart $Y$, Fierer N. The ecology of the phyllosphere: Geographic and phylogenetic variability in the distribution of bacteria on tree leaves. Environmental Microbiology. 2010;12(11):2885-93.

7. Rastogi G, Sbodio A, Tech JJ, Suslow TV, Coaker GL, Leveau JH. Leaf microbiota in an agroecosystem: Spatiotemporal variation in bacterial community composition on fieldgrown lettuce. The ISME Journal. 2012;6(10):1812.

8. Beattie GA, Lindow SE. Bacterial colonization of leaves: A spectrum of strategies. Phytopathology. 1999;89(5):353-9.

9. Agler MT, Ruhe J, Kroll S, Morhenn C, Kim S-T, Weigel D, et al. Microbial hub taxa link host and abiotic factors to plant microbiome variation. PLoS Biology. 2016;14(1):e1002352.

10. Laforest-Lapointe I, Messier C, Kembel SW. Tree phyllosphere bacterial communities: Exploring the magnitude of intra-and inter-individual variation among host species. PeerJ. 2016;4:e2367.

11. Monier J-M, Lindow S. Frequency, size, and localization of bacterial aggregates on bean leaf surfaces. Appl Environ Microbiol. 2004;70(1):346-55.

12. Tecon R, Leveau JH. The mechanics of bacterial cluster formation on plant leaf surfaces as revealed by bioreporter technology. Environmental Microbiology. 2012;14(5):1325-32.

13. Remus-Emsermann MN, Lücker S, Müller DB, Potthoff E, Daims H, Vorholt JA. Spatial distribution analyses of natural phyllosphere-colonizing bacteria on A rabidopsis thaliana revealed by fluorescence in situ hybridization. Environmental Microbiology. 2014;16(7):2329-40.

14. Morris CE, Monier J, Jacques M. Methods for observing microbial biofilms directly on leaf surfaces and recovering them for isolation of culturable microorganisms. Appl Environ Microbiol. 1997;63(4):1570-6.

15. Esser DS, Leveau JH, Meyer KM, Wiegand K. Spatial scales of interactions among bacteria and between bacteria and the leaf surface. FEMS Microbiology Ecology. 2015;91(3):fiu034.

16. Remus-Emsermann MN, Schlechter RO. Phyllosphere microbiology: At the interface between microbial individuals and the plant host. New Phytologist. 2018;218(4):1327-33.

17. Monier J-M, Lindow S. Spatial organization of dual-species bacterial aggregates on leaf surfaces. Appl Environ Microbiol. 2005;71(9):5484-93.

18. Peredo EL, Simmons SL. Leaf-FISH: Microscale imaging of bacterial taxa on phyllosphere. Frontiers in Microbiology. 2018;8:2669.

19. Remus-Emsermann MN, Tecon R, Kowalchuk GA, Leveau JH. Variation in local carrying capacity and the individual fate of bacterial colonizers in the phyllosphere. The ISME Journal. 2012;6(4):756. 
20. Remus-Emsermann MN, Kowalchuk GA, Leveau JH. Single-cell versus populationlevel reproductive success of bacterial immigrants to pre-colonized leaf surfaces. Environmental Microbiology Reports. 2013;5(3):387-92.

21. Monier J-M, Lindow S. Aggregates of resident bacteria facilitate survival of immigrant bacteria on leaf surfaces. Microbial Ecology. 2005;49(3):343-52.

22. Poza-Carrion C, Suslow T, Lindow S. Resident bacteria on leaves enhance survival of immigrant cells of Salmonella enterica. Phytopathology. 2013;103(4):341-51.

23. Grinberg M, Orevi T, Kashtan N. Bacterial surface colonization, preferential attachment and fitness under periodic stress. PLoS Computational Biology. 2019;15(3):e1006815.

24. Beattie GA, Lindow SE. Comparison of the behavior of epiphytic fitness mutants of Pseudomonas syringae under controlled and field conditions. Appl Environ Microbiol. 1994;60(10):3799-808.

25. Loper JE, Lindow SE. Lack of evidence for the in situ fluorescent pigment production by Pseudomonas syringae pv. syringae on bean leaf surfaces. J Phytopathology. 1987;77(10):1449-54.

26. Wilson M, Hirano S, Lindow S. Location and survival of leaf-associated bacteria in relation to pathogenicity and potential for growth within the leaf. Appl Environ Microbiol. 1999;65(4):1435-43.

27. Stockwell V, Johnson K, Sugar D, Loper J. Control of fire blight by Pseudomonas fluorescens A506 and Pantoea vagans C9-1 applied as single strains and mixed inocula. Phytopathology. 2010;100(12):1330-9.

28. Wilson M, Lindow S. Interactions between the biological control agent Pseudomonas fluorescens A506 and Erwinia amylovora in pear blossoms. Phytopathology. 1993;83(1):11723.

29. Choi K-H, Schweizer HP. mini-Tn7 insertion in bacteria with single attTn7 sites: Example Pseudomonas aeruginosa. Nature Protocols. 2006;1(1):153.

30. Morris CE, Monier J-M, Jacques M-A. A technique to quantify the population size and composition of the biofilm component in communities of bacteria in the phyllosphere. Appl Environ Microbiol. 1998;64(12):4789-95.

31. Franklin RB, Mills AL. The spatial distribution of microbes in the environment: Springer; 2007.

32. Daims H, Lücker S, Wagner M. Daime, A novel image analysis program for microbial ecology and biofilm research. Environmental Microbiology. 2006;8(2):200-13.

33. Daims $\mathrm{H}$, Wagner $\mathrm{M}$. In situ techniques and digital image analysis methods for quantifying spatial localization patterns of nitrifiers and other microorganisms in biofilm and flocs. Methods in Enzymology. 496: Elsevier; 2011. p. 185-215.

34. Reed M, Howard C. Stereological estimation of covariance using linear dipole probes. Journal of Microscopy. 1999;195(Pt 2):96-103.

35. Van Der Wal A, Tecon R, Kreft J-U, Mooij WM, Leveau JH. Explaining bacterial dispersion on leaf surfaces with an individual-based model (PHYLLOSIM). PloS One. 2013;8(10):e75633.

36. Schmidt $H$, Nunan N, Höck A, Eickhorst T, Kaiser C, Woebken D, et al. Recognizing patterns: Spatial analysis of observed microbial colonization on root surfaces. Front Environ Sci. 2018;6:61.

37. Gantner S, Schmid M, Dürr C, Schuhegger R, Steidle A, Hutzler P, et al. In situ quantitation of the spatial scale of calling distances and population density-independent $\mathrm{N}$ acylhomoserine lactone-mediated communication by rhizobacteria colonized on plant roots. FEMS Microbiology Ecology. 2006;56(2):188-94. 
38. Schillinger C, Petrich A, Lux R, Riep B, Kikhney J, Friedmann A, et al. Co-localized or randomly distributed? Pair cross correlation of in vivo grown subgingival biofilm bacteria quantified by digital image analysis. PLoS One. 2012;7(5).

39. Zhao K, Tseng BS, Beckerman B, Jin F, Gibiansky ML, Harrison JJ, et al. Psl trails guide exploration and microcolony formation in early P. aeruginosa biofilms. Nature.

2013;497(7449):388.

40. Hödl I, Hödl J, Wörman A, Singer G, Besemer K, Battin TJ. Voronoi tessellation captures very early clustering of single primary cells as induced by interactions in nascent biofilms. PloS One. 2011;6(10):e26368.

41. Mittelviefhaus M, Müller DB, Zambelli T, Vorholt JA. A modular atomic force microscopy approach reveals a large range of hydrophobic adhesion forces among bacterial members of the leaf microbiota. The ISME Journal. 2019;13(7):1878-82.

42. Laganenka L, Colin R, Sourjik V. Chemotaxis towards autoinducer 2 mediates autoaggregation in Escherichia coli. Nature Communications. 2016;7:12984.

43. Laganenka L, Sourjik V. Autoinducer 2-dependent Escherichia coli biofilm formation is enhanced in a dual-species coculture. Appl Environ Microbiol. 2018;84(5):e02638-17.

44. Dulla G, Lindow SE. Quorum size of Pseudomonas syringae is small and dictated by water availability on the leaf surface. Proceedings of the National Academy of Sciences. 2008;105(8):3082-7.

45. Bertsche U, Mayer C, Götz F, Gust AA. Peptidoglycan perception-sensing bacteria by their common envelope structure. International Journal of Medical Microbiology. 2015;305(2):217-23.

46. Monier J-M, Lindow S. Differential survival of solitary and aggregated bacterial cells promotes aggregate formation on leaf surfaces. Proceedings of the National Academy of Sciences. 2003;100(26):15977-82.

47. Grinberg M, Orevi T, Steinberg S, Kashtan N. Bacterial survival in microscopic droplets. bioRxiv. 2019.

48. Shank EA, Kolter R. New developments in microbial interspecies signaling. Current Opinion in Microbiology. 2009;12(2):205-14.

49. Nesli T, Orevi T, Grinberg M, Kastan N, Hadar Y, D M. Plant host affects bacteriabacteria interactions. In preparation. 2019. 